\title{
Density of Vacuum-Like Plasma and Hubble Constant
}

\author{
Ilya A. Obukhov \\ Research \& Development Company "System Resources”, Moscow, Russia \\ Email: i_obukhov@systemres.ru
}

How to cite this paper: Obukhov, I.A. (2017) Density of Vacuum-Like Plasma and Hubble Constant. Journal of High Energy Physics, Gravitation and Cosmology, 3, 572-587.

https://doi.org/10.4236/jhepgc.2017.34044

Received: August 5, 2017

Accepted: August 27, 2017

Published: August 30, 2017

Copyright (C) 2017 by author and Scientific Research Publishing Inc. This work is licensed under the Creative Commons Attribution International License (CC BY 4.0).

http://creativecommons.org/licenses/by/4.0/ (c) (i) Open Access

\begin{abstract}
The model in which expansion of the Universe leads to a generation of non-equilibrium vacuum-like electron-positron plasma is proposed and researched. The formulas that relate the Hubble's constant with the concentration of plasma particles and the cosmological constant are obtained. The collective properties of vacuum-like plasma are investigated. It is shown, that the coefficient of a two-photon annihilation in such plasma is nine times less than for the free particles. A simple formula for dark energy density as a function of electron mass and charge is obtained. It was demonstrated that acceleration of plasma's chemical potential fluctuations flow proportional of dark energy density.
\end{abstract}

\section{Keywords}

Non-Equilibrium Vacuum-Like Plasma, Hubble Constant, Dark Energy, Electron, Positron, Annihilation, Zero Enthalpy

\section{Introduction}

As shown in the article [1], under certain conditions electron-positron plasma has the zero density of an enthalpy. For this plasma the ratio is fair

$$
\varepsilon=-p,
$$

where $\varepsilon$ is the energy density and $p$ is the pressure.

Unusual properties of such material medium are caused by the existence of the random electromagnetic field generated by transitions between various quantum states of electrons and positrons. If a constant temperature $T$ satisfies the conditions

$$
p_{F}^{(e) 2} / m \ll k_{B} T \ll m c^{2}, p_{F}^{(h) 2} / m \ll k_{B} T \ll m c^{2},
$$


the energy density $\varepsilon^{(s)}$ and pressure $p^{(s)}$ of a random electromagnetic field are related to the temperature by the formulas

$$
\begin{gathered}
\varepsilon^{(s)} \approx-\pi^{-2}(4 / \pi-1)\left(e^{\left.2 m k_{B} T / \hbar^{2}\right)^{2},}\right. \\
p^{(s)}=-\varepsilon^{(s)} / 3 \approx\left(3 \pi^{2}\right)^{-1}(4 / \pi-1)\left(e m k_{B} T / \hbar^{2}\right)^{2},
\end{gathered}
$$

where $p_{F}^{(e)}$ and $p_{F}^{(h)}$ are the Fermi momenta of electrons and positrons; $m$ and $e$ are the mass and the electric charge of the electron; $c$ is the velocity of light in vacuum; $k_{B}$ is the Boltzmann's constant; $\hbar$ is the Planck's constant divided by $2 \pi$.

In order that plasma with such properties is vacuum-like [2], it is necessary to impose the additional requirement of electro-neutrality [3]. In this case, the plasma becomes non-equilibrium. The sum of chemical potentials of the electrons $F^{(e)}$ and of the positrons $F^{(h)}$ is equal to zero

$$
F^{(e)}+F^{(h)}=0
$$

and their difference is expressed by the ratio

$$
F^{(e)}-F^{(h)}=2 c\left(m^{2} c^{2}+p_{F}^{2}\right)^{1 / 2} \approx 2 m c^{2}\left[1+(1 / 2)\left((4 / \pi-1) \alpha\left(k_{B} T / m c^{2}\right)^{2}\right)^{2 / 3}\right],
$$

where $\alpha=e^{2} / \hbar c$ is the fine-structure constant approximately equal to $1 / 137$. In this case, Fermi momenta of electrons and positrons

$$
\begin{gathered}
p_{F}^{(e)}=c^{-1} \theta\left(\left(F^{(e)}-m c^{2}\right) / m c^{2}\right)\left(F^{(e) 2}-m^{2} c^{4}\right)^{1 / 2}, \\
p_{F}^{(h)}=c^{-1} \theta\left(\left(-F^{(h)}-m c^{2}\right) / m c^{2}\right)\left(F^{(h) 2}-m^{2} c^{4}\right)^{1 / 2},
\end{gathered}
$$

are equal

$$
p_{F}^{(e)}=p_{F}^{(h)}=p_{F},
$$

and are connected with temperature by the equation

$$
p_{F}^{3}=(4 / \pi-1)\left(e^{2} k_{B}^{2} T^{2} m / \hbar c^{2}\right) .
$$

Equation (4.5) is the consequence of the relation (1).

The electron-positron plasma, which satisfies the conditions (4.1)-(4.5), together with random electromagnetic field, is the vacuum-like material medium that has a zero enthalpy

$$
h=p+\varepsilon=0,
$$

and zero entropy

$$
s=T^{-1}\left(\varepsilon+p-F^{(e)} n^{(e)}-F^{(h)} n^{(h)}\right)=0
$$

too. Due to these properties, it can play the role of dark energy. However, the energy density of researched medium is negative and its pressure is positive.

The total energy density and pressure are expressed in the following form:

$$
\varepsilon=-p \approx-m c^{2} n^{(\Lambda)} .
$$


For the energy densities of electrons, positrons and random electromagnetic field the formulas are true

$$
\varepsilon^{(e)} \approx m c^{2} n^{(\Lambda)}, \varepsilon^{(h)} \approx m c^{2} n^{(\Lambda)}, \varepsilon^{(s)} \approx-3 m c^{2} n^{(\Lambda)} .
$$

where

$$
n^{(\Lambda)}=n^{(e)}=n^{(h)}=(8 \pi / 3)\left(p_{F} / 2 \pi \hbar\right)^{3}=\left(3 \pi^{2}\right)^{-1}(4 / \pi-1) m\left(e k_{B} T / \hbar^{2} c\right)^{2},
$$

$n^{(e)}$ and $n^{(h)}$ are the concentrations of electrons and positrons. If to assume that absolute value of plasma's energy density is equal to the density of dark energy, the following estimations can be obtained: $n^{(\Lambda)} \approx 7.92 \times 10^{-3} \mathrm{~cm}^{-3}$, $T \approx 1.54 \times 10^{-5} \mathrm{~K}, \quad p_{F} \approx 6.50 \times 10^{-28} \mathrm{~g} \cdot \mathrm{cm} \cdot \mathrm{s}^{-1}$ [1].

If the electro-neutral plasma is in the state of chemical equilibrium, the following condition

$$
F^{(e)}(x)=F^{(h)}(x)
$$

is satisfied. Then the equality to zero of chemical potentials of electrons and positrons follows from the relationship (4.1) and equality to zero of their Fermi momenta from the relationship (4.3) follows too. If the density of plasma's enthalpy is equal to zero then the relationship (4.5) is satisfied. In the case of the equilibrium state of plasma, Fermi momenta, the temperature, energy density, pressure, electron concentration and positron concentration are equal to zero. It means that vacuum-like plasma does not exist in an equilibrium state.

Thus, the necessary condition of existence of the vacuum-like electron-positron plasma is chemical non-equilibrium. The possible causes of non-equilibrium demand the clarification. The present article is devoted to this problem.

\section{Equations of Particles Balance in Non-Equilibrium Plasma}

The equations of electrons and positrons balance may be written in the following form [4] [5]

$$
\begin{aligned}
& \partial_{\mu} j^{(e) \mu}(x)=\partial n^{(e)}(x) / \partial t+\left(\nabla, j^{(e)}(x)\right)=g(x), \\
& \partial_{\mu} j^{(h) \mu}(x)=\partial n^{(h)}(x) / \partial t+\left(\nabla, j^{(h)}(x)\right)=g(x),
\end{aligned}
$$

where $j^{(e) \mu}(x)=\left(c n^{(e)}(x), \boldsymbol{j}^{(e)}(x)\right)$ and $j^{(h) \mu}(x)=\left(c n^{(h)}(x), \boldsymbol{j}^{(h)}(x)\right)$ are the flows density of electrons and of positrons; $x=(c t, \boldsymbol{r}) ; t$ is a time and $\boldsymbol{r}$ is a vector of spatial coordinates; $n^{(e)}(x), n^{(h)}(x)$ and $\boldsymbol{j}^{(e)}(x), \boldsymbol{j}^{(h)}(x)$ are the concentrations and spatial flows density of electrons and positrons; $g(x)$ is the velocity of the generation and of the annihilation of particle-antiparticle pairs.

In the absence of external electromagnetic fields and the constant temperature density flow, expressions are true

$$
\begin{aligned}
& j^{(e) \mu}(x)=c(2 \pi \hbar)^{-3} \sum_{r} \int\left(\mathrm{d}^{3} k / k_{0}\right) k^{\mu} f\left(s^{(e)}\left(F^{(e)}(x), T, U^{(e) \mu}(x), k\right)\right), \\
& j^{(h) \mu}(x)=c(2 \pi \hbar)^{-3} \sum_{r} \int\left(\mathrm{d}^{3} k / k_{0}\right) k^{\mu} f\left(s^{(h)}\left(F^{(h)}(x), T, U^{(h) \mu}(x), k\right)\right) .
\end{aligned}
$$


Here $k^{\mu}$ is electron momentum four-vector or positron momentum four-vector for which the zero component is represented in the form

$$
\begin{gathered}
k_{0}=\left(m^{2} c^{2}+k^{2}\right)^{1 / 2}>0, k^{2}=(\boldsymbol{k}, \boldsymbol{k}), \\
f\left(s^{(e, h)}\right)=\left(1+\exp \left\{s^{(e, h)}\right\}\right)^{-1}
\end{gathered}
$$

is the Fermi-Dirac distribution function;

$$
s^{(e)}=\left(k_{\mu} U^{(e) \mu}-F^{(e)}\right) / k_{B} T, s^{(h)}=\left(k_{\mu} U^{(h) \mu}+F^{(h)}\right) / k_{B} T,
$$

$U^{(e) \mu}$ and $U^{(h) \mu}$ are the hydrodynamic velocities of particles and antiparticles [4], for which the relations are satisfied

$$
U^{(e) \mu} U_{\mu}^{(e)}=c^{2}, U^{(h) \mu} U_{\mu}^{(h)}=c^{2}, U_{0}^{(e)}>0, U_{0}^{(h)}>0 .
$$

The left parts of Equations (7.1) and (7.2) depend on coordinates and time using chemical potentials $F^{(e)}(x), F^{(h)}(x)$ and using hydrodynamic velocities $U^{(e) \mu}(x), U^{(h) \mu}(x)$. Spatial components of hydrodynamic velocities may be defined by [5] [6].

$$
\boldsymbol{U}^{(e)}(x)=-\left(\tau^{(e)} / m\right) \nabla F^{(e)}(x), \boldsymbol{U}^{(h)}(x)=\left(\tau^{(h)} / m\right) \nabla F^{(h)}(x) .
$$

Components $U^{(e) 0}(x)$ and $U^{(h) 0}(x)$ are calculated from the expressions (9.3). Times of momentum relaxation for electron $\tau^{(e)}$ and for positron $\tau^{(h)}$ are positive values. They define the electric resistance of medium [6] [7]. Their physical nature and numerical values are determined by stochastic scattering of electrons and positrons [7].

Taking into account definitions (10) left parts of the Equations (7.1) and (7.2) depend on the coordinates and time only through the chemical potentials and their derivatives. Electrons and positrons interact with each other by emitting and absorbing electromagnetic fields. As a result at a constant temperature and under the condition of absence of external interaction, the Equations (7.1) and (7.2) must describe the relaxation of the system to the state of the local chemical equilibrium, which is determined by equality (6). If chemical potentials do not depend on coordinates and time, global chemical equilibrium reached.

Using the analogy with the theory of charge transport in mesoscopic structures we will assume that $g(x)$ is a function of the difference between the chemical potentials of electrons and positrons, and $g(x)$ equal zero in state of chemical equilibrium. We will choose this function in the form

$$
g(x)=-c^{-2} \omega^{(e, h)} \dot{i}^{(e)}(x) i^{(h)}(x)\left(1-\exp \left\{-\left(F^{(e)}(x)-F^{(h)}(x)\right) / k_{B} T\right\}\right),
$$

where

$$
\begin{aligned}
& i^{(e)}(x)=m c^{2}(2 \pi \hbar)^{-3} \sum_{r} \int\left(\mathrm{d}^{3} k / k_{0}\right) f\left(s^{(e)}\left(F^{(e)}(x), T, U^{(e) \mu}(x), k\right)\right), \\
& i^{(h)}(x)=m c^{2}(2 \pi \hbar)^{-3} \sum_{r} \int\left(\mathrm{d}^{3} k / k_{0}\right) f\left(s^{(h)}\left(F^{(h)}(x), T, U^{(h) \mu}(x), k\right)\right)
\end{aligned}
$$

are the invariant density of electrons and positrons; $\omega^{(e, h)}$ is the coefficient of 
two-photon annihilation of electron and positron, its dimension is $\mathrm{cm}^{3} \cdot \mathrm{c}^{-1}$. Such choosing of function $g(x)$ provides a relaxation of solutions of Equations (7.1) and (7.2) to the state of local chemical equilibrium (6).

The characteristic relaxation times of the concentration fluctuations determined by expressions

$$
\tau_{n e}=c^{2} n^{(e)} / Y \omega^{(e, h)} \boldsymbol{i}^{(e)} \boldsymbol{i}^{(h)}, \tau_{n h}=c^{2} n^{(h)} / Y \omega^{(e, h)} \boldsymbol{i}^{(e)} \boldsymbol{i}^{(h)},
$$

where indicated

$$
Y=1-\exp \left\{-\left(F^{(e)}-F^{(h)}\right) / k_{B} T\right\} .
$$

For relaxation time of chemical potentials difference the formula are fair

$$
\tau_{r e l}=c^{2} n^{(e)} n^{(h)} /\left(n^{(e)}+n^{(h)}\right) \omega^{(e, h)} i^{(e)} \boldsymbol{i}^{(h)} .
$$

By using, the definition (10) can be obtained the expression for characteristic distance of relaxation to zero of the difference of the chemical potentials

$$
L_{\text {rel }}=c\left[\tau^{(e)} \tau^{(h)} n^{(e)} n^{(h)} k_{B} T / m\left(\tau^{(e)} n^{(e)}+\tau^{(h)} n^{(h)}\right) \omega^{(e, h)} i^{(e)} i^{(h)}\right]^{1 / 2} .
$$

In researched vacuum-like plasma when the relationships

$$
i^{(e)}=i^{(h)} \approx c n^{(e)}=c n^{(h)}=c n^{(\Lambda)}
$$

are fair we obtain

$$
\begin{gathered}
\tau_{n e}=\tau_{n h}=\tau^{(\Lambda)}=1 / Y \omega^{(e, h)} n^{(\Lambda)}, \\
\tau_{\text {rel }}=Y \tau^{(\Lambda)} / 2, \\
L_{\text {rel }}=\left(\hbar \tau^{(\Lambda)} Y / 2 m\right)^{1 / 2} .
\end{gathered}
$$

The Formula (16.3) was obtained from the expression (15) with assumption that

$$
\tau^{(e)}=\tau^{(h)}=\tau_{T}=\hbar / k_{B} T .
$$

We will use the formula for a cross-section of two-photon annihilation that was obtained by Dirac at 1930 [8] for estimations. It follows that the expression for coefficient

$$
\omega^{(e, h)} \sim \omega_{0}^{(e, h)}=c \pi r_{0}^{2},
$$

where

$$
r_{0}=e^{2} / m c^{2}
$$

is the classical radius of the electron, which is approximately equal to $2.81 \times 10^{-13} \mathrm{~cm}$. The corresponding estimation for the coefficient $\omega_{0}^{(e, h)} \approx 7.42 \times 10^{-15} \mathrm{~cm}^{3} \cdot \mathrm{s}^{-1}$ is correct. According to Dirac [8] this result is true if we neglect the interaction between particles and antiparticles.

Using Formulas (18.1) and (18.2) from expressions (16.1)-(16.3) we will obtain

$$
\tau^{(\Lambda)}=2 \tau_{r e l} \sim\left(1 / \pi c n^{(\Lambda)}\right)\left(m c^{2} / e^{2}\right)^{2}=\tau_{c}\left(3 \pi / \alpha^{2}\right)\left(m c / p_{F}\right)^{3},
$$




$$
L_{r e l}^{2} \sim L_{c}^{2}\left(3 \pi / 2 \alpha^{2}\right)\left(m c / p_{F}\right)^{3},
$$

where

$$
\begin{gathered}
\tau_{c}=\hbar / m c^{2}, \\
L_{c}=\left(\hbar \tau_{c} / m\right)^{1 / 2}=\hbar / m c .
\end{gathered}
$$

$L_{c}$ Time $\tau_{c}$ is approximately equal to $1.28 \times 10^{-21} \mathrm{~s}$ and Compton wavelength is about $3.86 \times 10^{-11} \mathrm{~cm}$. According to paper [1] the estimations

$$
n^{(\Lambda)} \sim 10^{-3} \mathrm{~cm}^{-3}, p_{F} \sim 10^{-28} \mathrm{~g} \cdot \mathrm{cm} / \mathrm{s}, T \sim 10^{-5} \mathrm{~K}
$$

is correct. It means

$$
\tau_{T} \sim 10^{-7} \mathrm{~s}, \tau^{(\Lambda)} \sim 10^{17} \mathrm{~s}, \tau_{\text {rel }} \sim 10^{17} \mathrm{~s}, L_{\text {rel }} \sim 10^{8} \mathrm{~cm} .
$$

Such big times $\tau_{n e}, \tau_{n h}, \tau_{\text {rel }}$ and diffusion length $L_{\text {rel }}$ are caused by a factor $\mathrm{mc} / \mathrm{p}_{\mathrm{F}} \sim 10^{10}$, i.e. by the low density of plasma.

The characteristic relaxation time of the concentration fluctuations is about the age of the Universe. The time of restoration of chemical equilibrium exceeds the age of the Universe by eight orders.

\section{Expansion of the Universe and Non-Equilibrium Plasma}

The obtained results show, that in normal scales of times small deviations from the state of chemical equilibrium of electron-positron plasma can be considered as stationary. However, it relates to small fluctuations for which the estimation is fair

$$
\delta n \ll n^{(\Lambda)} \Rightarrow \delta\left(F^{(e)}-F^{(h)}\right) \sim \delta\left(p_{F}^{2} / 2 m\right) \ll k_{B} T \ll m c^{2} .
$$

The presence and long time existence of such fluctuations can't be the cause of high level of non-equilibrium plasma

$$
\delta\left(F^{(e)}-F^{(h)}\right) \sim m c^{2},
$$

defined by Formula (4.2)

Various external influence scan be sources of non-equilibrium plasma. For example, it may be the external electromagnetic radiation, current flow through the regions with high gradients of concentrations of plasma particles [6], the dependence of concentration on time caused by the external reasons. The last hypothesis seems to be the most realistic.

According to the modern vision, in the current period of the cosmic history, the spatial size of the Universe increases as a function of time. The rate of this expansion is measured by Hubble's constant $H \approx 2.20 \times 10^{-18} \mathrm{~s}^{-1}$. We assume that the number of electrons and equal number of positrons of the researched plasma $N$ do not depend from space and time coordinates. In this case, the expansion of the Universe must lead to a change of particles' concentration due to the increase of volume $V$ occupied by electrons and positrons.

$$
\partial n^{(e)} / \partial t=-n^{(e)}\left(V^{-1} \partial V / \partial t\right)=-3 n^{(e)} H, \partial n^{(h)} / \partial t=-n^{(h)}\left(V^{-1} \partial V / \partial t\right)=-3 n^{(h)} H .
$$


Taking into account the weak dependence of $H$ from time, generated by sources (21) non-equilibrium may be considered practically stationary.

From the Equations (7.1), (7.2) and the Formula (21) for vacuum-like plasma we obtain

$$
\begin{aligned}
& n^{(e)}=n^{(h)}=n^{(\Lambda)}, \\
& Y=3 H / \omega^{(e, h)} n^{(\Lambda)} .
\end{aligned}
$$

According to the expression (4.2) $Y$ exceeds a unit at a size about $\exp \left\{-10^{14}\right\}$, then we will find plasma density from the Formula (22.2)

$$
n^{(\Lambda)} \approx 3 H / \omega^{(e, h)} .
$$

If to use $\omega_{0}^{(e, h)}$ defined by the expression $(18.1)$ as coefficient $\omega^{(e, h)}$, then from relationship (23) we obtain

$$
\rho^{(\Lambda)}=-m n^{(\Lambda)} \approx-8.09 \times 10^{-31} \mathrm{~g} \cdot \mathrm{cm}^{-3} .
$$

The absolute value of this density is about nine time less than experimental result $\rho_{\mathrm{exp}}^{(\Lambda)} \approx(7.21 \pm 0.25) \times 10^{-30} \mathrm{~g} \cdot \mathrm{cm}^{-3} \quad$ [9].

The electron-positron annihilation probability defined by the Formulas (18.1) and (18.2) doesn't consider the additional interaction between particles caused by random electromagnetic field and collective properties of plasma. In Introduction the formulas for the energy density of random electromagnetic field are presented. They include energy density of random field oneself and energy density of its interaction with electrons and positrons too.

The energy-momentum tensor of a random electromagnetic field, which includes the interaction with particles and antiparticles, can be represented in the form [1]

$$
\begin{gathered}
t^{\mu v}=t^{\mu v}(A(x))+t^{\mu v}(q(x), A(x)), \\
t^{\mu v}(A(x))=-(4 \pi c)^{-1}\left(F^{\mu k}(x) F^{v}{ }_{k}(x)-(1 / 4) \eta^{\mu v} F^{k l}(x) F_{k l}(x)\right), \\
t^{\mu v}(q(x), A(x))=-\left(e / c^{2}\right) q^{\mu}(x) A^{v}(x) .
\end{gathered}
$$

Here $A^{\mu}(x)$ is a vector-potential of a random electromagnetic field;

$$
F^{\mu v}(x)=\partial^{\mu} A^{v}(x)-\partial^{v} A^{\mu}(x)
$$

is a tensor of this field; $\eta^{\mu \nu}$ is Minkowski tensor;

$$
q^{\mu}(x)=q^{(e) \mu}(x)-q^{(h) \mu}(x)
$$

is a difference between flows of electrons $q^{(e) \mu}(x)$ and positrons $q^{(h) \mu}(x)$.

Energy density and pressure of random electromagnetic field are calculated from relationships

$$
\varepsilon^{(s)}=c T^{00}, p^{(s)}=-(c / 3)\left(T_{\mu}^{\mu}-T_{0}^{0}\right),
$$

where

$$
T^{\mu v}=\left\langle t^{\mu v}\right\rangle
$$


is a tensor averaged by random processes of transitions between states of particle-particle, antiparticle-antiparticle, particle-antiparticle.

The tensor $T^{\mu v}$ according to the Formula (24.1) may be represented by the sum

$$
T^{\mu v}=T^{(A) \mu v}+T^{(\mathrm{int}) \mu v}
$$

where

$$
T^{(A) \mu v}=\left\langle t^{\mu v}(A(x))\right\rangle, T^{(\mathrm{int}) \mu v}=\left\langle t^{\mu v}(q(x), A(x))\right\rangle,
$$

are the average energy-momentum tensor of the electromagnetic field and of it's interaction with electrons and positrons.

Using the relationships (25.1), (25.2) and (26.1), (26.2) it is possible to extract density of electromagnetic interaction potential from total expressions [10]

$$
v=c T_{\mu}^{(\mathrm{int}) \mu} \text {. }
$$

Considering that

$$
T_{\mu}^{(A) \mu} \equiv 0
$$

we will find

$$
v=\varepsilon^{(s)}-3 p^{(s)}
$$

The density of potential $v$ defines the contribution of a random electromagnetic field to the scattering matrix of spinor particles [10].

Energy density and pressure of vacuum-like plasma may be represented in the form

$$
\begin{gathered}
\varepsilon^{(s)}=\varepsilon^{(e, e)}+\varepsilon^{(h, h)}+\varepsilon^{(e, h)}, \\
p^{(s)}=p^{(e, e)}+p^{(h, h)}+p^{(e, h)}
\end{gathered}
$$

where

$$
\begin{gathered}
\varepsilon^{(e, e)}=-3 p^{(e, e)}=-3 m c^{2} n^{(\Lambda)} / 2, \\
\varepsilon^{(h, h)}=-3 p^{(h, h)}=-3 m c^{2} n^{(\Lambda)} / 2, \\
\varepsilon^{(e, h)}=p^{(e, h)}=-4 \pi\left(e \hbar n^{(\Lambda)} / m c\right)^{2}
\end{gathered}
$$

are the contributions of transitions of electron-electron, positron-positron, electron-positron. The main contribution to the total energy density and the total pressure of a random electromagnetic field give the electron-electron and positron-positron transitions [1]. Evaluations follow from Formulas (28.3)-(28.5)

$$
\left|\varepsilon^{(e, h)} / \varepsilon^{(s)}\right| \sim\left|p^{(e, h)} / p^{(s)}\right| \sim \alpha\left(p_{F} / m c\right)^{3} \ll 1
$$

According to the structure of relationships (28.1) and (28.2) from (27.2), we obtain

$$
\begin{gathered}
v=v^{(e, e)}+v^{(h, h)}+v^{(e, h),} \\
v^{(e, e)}=v^{(h, h)}=-3 m c^{2} n^{(\Lambda)},
\end{gathered}
$$




$$
v^{(e, h)}=8 \pi\left(e \hbar n^{(\Lambda)} / m c\right)^{2}
$$

These expressions demonstrate that the attraction between particles with an identical sign of the electric charge is the result of random electron-electron and positron-positron transitions. The density of attraction potential is three times more than energy density of particles.

Random electrons-positrons transitions lead to repulsion between particles with different sign of the electric charge. The density of repulsion potential is much less than the energy density of plasma components.

Strong attractive interaction must lead to unusual properties of electrons and positrons gasses. For example, for researched environment the characteristic distance can be defined

$$
r_{e q}=e^{2} / 3 m c^{2}
$$

at which the attraction between electron and electron or between positron and positron is balanced by Coulomb repulsion between these particles. The repulsion dominates at distances less than $r_{e q}$. The attraction dominates at distances more than $r_{e q}$. i.e. $r_{e q} \approx 9.36 \times 10^{-14} \mathrm{~cm}$ defines the distance of stable equilibrium.

The characteristic distance

$$
r_{\text {run }}=\left(1 / 8 \pi n^{(\Lambda)}\right)(m c / \hbar)^{2}=(3 \pi / 8)\left(m c / p_{F}\right)^{2}\left(\hbar / p_{F}\right)
$$

may be defined for interaction between electrons and positrons too. This is the distance at which force of Coulomb attraction force is equal to the force of repulsion force caused by random transitions. Distance $r_{r u n}$ is huge, it is about $3.36 \times 10^{21} \mathrm{~cm}$. Ratio of distances $r_{\text {run }} / r_{\text {eq }}$ is huge too-about $10^{34}$. Physical interpretations of distance $r_{e q}$ and distance $r_{r u n}$ are different. The distance $r_{r u n}$ corresponds to the boundary of a region outside of which electrons and positrons run up.

The distance $r_{e q}$ is exactly three times less than the classical radius of electron defined by Formula (18.2). A simple replacement $r_{0}$ to $r_{e q}$ leads to "proper" density value $\rho^{(\Lambda)}=-7.28 \times 10^{-30} \mathrm{~g} \cdot \mathrm{cm}^{-3}$. But such simple change needs reasoning.

According to the condensed matter theory [11] [12] the Heisenberg operators of electron component $\Psi^{(e)}(x)$ and positron component $\Psi^{(h)}(x)$ of vacuum-like plasma are solutions of the equations

$$
\begin{aligned}
& \left(i \hbar c \gamma^{\mu} \partial_{\mu}-m c^{2}+F^{(e)}+V^{(e)}\right) \Psi^{(e)}(x)=0, \\
& \left(i \hbar c \gamma^{\mu} \partial_{\mu}-m c^{2}-F^{(h)}-V^{(h)}\right) \Psi^{(h)}(x)=0 .
\end{aligned}
$$

Here $\gamma^{\mu}$ are the Dirac matrix and

$$
V^{(e)}=\left(v^{(e, e)}+v^{(e, h)}\right) / n^{(\Lambda)}, V^{(h)}=\left(v^{(h, h)}+v^{(e, h)}\right) / n^{(\Lambda)} .
$$

Using the Expressions (4.1), (4.2) and (29.1), (29.2), (29.3) from Equations (32.1) and (32.2) we will obtain the approximate equations 


$$
\begin{aligned}
& \left(i \hbar c \gamma^{\mu} \partial_{\mu}-3 m c^{2}\right) \Psi^{(e)}(x)=0, \\
& \left(i \hbar c \gamma^{\mu} \partial_{\mu}+3 m c^{2}\right) \Psi^{(h)}(x)=0 .
\end{aligned}
$$

Expressions (34.1) and (34.2) describe the quasiparticles of researched plasma. They are equal to the equations for a usual free electron and free positron operators in which the electron mass $m$ is replaced to effective mass equal $3 m$. If to use the solutions of Equations (34.1) and (34.2) with positive energy for description of quasiparticles states and for construction of causal Green function we will obtain the well known expression for cross section of two-photon quasiparticles annihilations [10] in which $m$ will replaced to $3 m$. In non-relativistic limit for probability of this process we will obtain the formula

$$
\omega^{(e, h)}=c \pi\left(e^{2} / 3 m c^{2}\right)^{2} .
$$

Exactly this result will be obtained if you make a change $r_{0} \rightarrow r_{e q}$ in Formula (18.1).

Using $\omega^{(e, h)}$ defined by Expression (35) from relationship (22.2) for non-equilibrium level of plasma we will obtain

$$
\begin{aligned}
Y & =81 \pi H m^{2} \hbar^{3} c^{3} / p_{F}^{3} e^{4}=\left(81 \pi / \alpha^{2}\right)\left(\hbar H / m c^{2}\right)\left(m c / p_{F}\right)^{3} \\
& =3 \pi(3 / \alpha)^{3}(4 / \pi-1)^{-1}\left(\hbar H / k_{B} T\right)\left(m c^{2} / k_{B} T\right) .
\end{aligned}
$$

Assuming that $Y \approx 1$ from Formulas (36), (23) and definitions of dark energy density $\rho^{(\Lambda)}$ and cosmological constant $\Lambda$ [3] we will find

$$
\begin{gathered}
T \approx\left(9 c / k_{B}\right)\left(\pi \hbar H m / \alpha^{3}(4 / \pi-1)\right)^{1 / 2}, \\
n^{(\Lambda)} \approx 27 \mathrm{Hm}^{2} c^{3} / \pi e^{4}, \\
\rho^{(\Lambda)}=-m n^{(\Lambda)} \approx-\left(H / \pi e^{4}\right)(3 m c)^{3}, \\
\Lambda=\rho^{(\Lambda)}\left(8 \pi G / c^{2}\right) \approx-216 G H c m^{3} / e^{4} .
\end{gathered}
$$

Here $G \approx 6.67 \times 10^{-8} \mathrm{~cm}^{3} \cdot \mathrm{g}^{-1} \cdot \mathrm{s}^{-2}$ is the Newtonian gravitation constant. From Expression (37.2) for concentration follows $n^{(\Lambda)} \approx 8.00 \times 10^{-3} \mathrm{~cm}^{-3}$ and from Formula (37.4) for cosmological constant follows $\Lambda \approx-1.36 \times 10^{-56} \mathrm{~cm}^{-2}$.

From the Formula (37.2) new representations for distance $r_{\text {run }}$ can be obtained

$$
r_{\text {run }}=\left(\alpha^{2} / 216\right)(c / H)=\left(\alpha^{2} / 216\right) r_{H}
$$

And the ratio of distance $r_{\text {run }}$ to distance $r_{e q}$

$$
r_{\text {run }} / r_{\text {eq }}=(\alpha / 72)\left(m c^{2} / \hbar H\right)=(\alpha / 72)\left(m / m_{H}\right),
$$

where $r_{H}=c / H \approx 1.36 \times 10^{28} \mathrm{~cm}$ is the distance which may be interpreted as the characteristic size of the Universe; $m_{H}=\left(\hbar H / c^{2}\right) \approx 2.57 \times 10^{-66} \mathrm{~g}$ is very small mass that was call the "Hubble mass" [13].

Obtained Formulas (37.1)-(37.4) and (38.1), (38.2) represent the characteristics of vacuum-like plasma as the functions of the fundamental constants and the 
mass of plasma components. It is interesting that obtained expressions for dark energy density and for cosmological constant do not include the Planck constant.

The Expression (37.1) for density of vacuum-like plasma can be represented in form

$$
n^{(\Lambda)} \approx 3 / \pi r_{H} r_{e q}^{2} .
$$

This representation is strong spatial anisotropic because $r_{e q} / r_{H} \sim 10^{-41}$. It corresponds to space in the form of a one-dimensional wire.

Thus, the expansion of the Universe must lead the electron-positron plasma to non-equilibrium state. Balance equations of plasma particles connect the concentrations of electrons and positrons with Hubble's constant.

\section{Plasma Fluctuations and Acceleration}

In frameworks of $\Lambda \mathrm{CDM}$ model of cosmology for acceleration of Universe expansion the formula is true

$$
\ddot{a} / a \approx\left(3 H^{2} / 2\right)\left(\Omega^{(\Lambda)}-1 / 3\right)
$$

where $a$ is the radius of the world and $\ddot{a}$ is its second derivative on time,

$$
\Omega^{(\Lambda)}=\rho^{(\Lambda)} / \rho^{(c)}
$$

and

$$
\rho^{(c)}=3 H^{2} / 8 G
$$

is the critical matter density in the Universe that approximately equal $8.67 \times 10^{-30} \mathrm{~g} \cdot \mathrm{cm}^{-3}$. According to modern interpretation of astrophysical data the dark energy density is $7.21 \times 10^{-30} \mathrm{~g} \cdot \mathrm{cm}^{-3}$ and we obtain

$$
\Omega^{(\Lambda)} \approx 0.83, \ddot{a} / a \approx 3.62 \times 10^{-36} \mathrm{~s}^{-2}
$$

that correspond to accelerated expansion of Universe.

For researched vacuum-like electron-positron plasma we obtain the another values

$$
\Omega^{(\Lambda)}=-72 G m^{3} c^{3} / H e^{4} \approx-0.84, \ddot{a} / a \approx-8.52 \times 10^{-36} \mathrm{~s}^{-2}
$$

that correspond to decelerated expansion of Universe. In all cases $|\ddot{a}| \sim 10^{-11} \mathrm{~cm} / \mathrm{s}^{-2}$ if $a$ is about megaparsec.

The positive density of dark energy follows from interpretation of astrophysical observations of supernovas Ia type in terms of $\Lambda \mathrm{CDM}$ model [14]. But it is not the only way of an interpretation of observed data. For instance, in article [15] was demonstrated that these observations may be interpreted in the terms of decelerated Universe expansion if assumed that in intergalactic space the index of light's refraction is about 1.50 .

The phenomenon of supernovas positive acceleration can be explained not only with the help of dark energy concept. This result may be obtained by the different versions of the Gravity Theory which are alternative to General Rela- 
tivity [16]. For example, it may be the model, in which the hypothetical massive classical gravitons are introduced [13].

In this context, the hypothesis of non-gravitational nature of supernovas Ia acceleration may be considered too. For example, this acceleration may be produced by fluctuations of vacuum-like plasma that were connected with supernova explosion. The fluctuations flow is the function of the time. It means that the fluctuations move with acceleration. Of course, the source of electromagnetic radiation in this case move with acceleration too.

Let's assume that fluctuations not destroy the plasma electroneutrality and condition

$$
F^{(e)}=-F^{(h)}=F
$$

is true. The chemical potential $F$ we will present in form

$$
F=F^{(\Lambda)}+F_{1}
$$

Where according expressions (5.3) and (37.2) $F^{(\Lambda)}$ satisfies of formula

$$
F^{(\Lambda)}=m c^{2}\left(1+\left(\left(81 \pi / \alpha^{2}\right)\left(\hbar H / m c^{2}\right)\right)^{2 / 3}\right)^{1 / 2}
$$

Value of $F^{(\Lambda)}$ corresponds to concentration $n^{(\Lambda)}$ of plasma particles. For $F_{1}$ from Equations (7.1) and (7.2) we obtain

$$
\begin{aligned}
& \partial F_{1} / \partial t-F^{-1}\left(F^{2}-m^{2} c^{4}\right)^{-1 / 2}(\tau / 3 m)\left(\nabla,\left(F^{2}-m^{2} c^{4}\right)^{3 / 2} \nabla F_{1}\right)=-\left(\omega^{(e, h)} Y / 9 \pi^{2} \hbar^{3} c^{3}\right) \\
& \left(\theta\left(\left(F-m c^{2}\right) / m c^{2}\right)\left(F^{2}-m^{2} c^{4}\right)^{5 / 2} / F-\left(F^{(\Lambda) 2}-m^{2} c^{4}\right)^{5 / 2} / F^{(\Lambda)}\right) .
\end{aligned}
$$

If fluctuations are small

$$
\left|F_{1}\right| \ll p_{F}^{2} / 2 m
$$

the simple equation is true

$$
\partial F_{1} / \partial t-D_{F} \Delta F_{1}=-5 H F_{1}
$$

where

$$
D_{F}=\left(p_{F}^{2} / m\right)(\tau / 3 m)
$$

is a coefficient of diffusion in vacuum-like electron-positron plasma.

For degenerate nonequilibrium plasma it is logical to assume that

$$
\tau=\tau_{p}=2 m \hbar / p_{F}^{2}=2\left(\hbar / m c^{2}\right)\left(\left(\alpha^{2} / 81 \pi\right)\left(m c^{2} / \hbar H\right)\right)^{2 / 3} .
$$

This value is about $4.52 \mathrm{~s}$. It is interesting, that value of $\tau_{p}$ correlates with the characteristic time of cosmic gamma-ray burst [17]. For $D_{F}$ we obtain

$$
D_{F}=(2 / 3)(\hbar / \mathrm{m}) \approx 0.77 \mathrm{~cm}^{2} \cdot \mathrm{s}^{-1} \text {. }
$$

From Equation (41) follows that the characteristic time of relaxation of plasma chemical potential in spatial independent case is

$$
\tau_{F}=1 / 5 H \approx 9.09 \times 10^{16} \mathrm{~s} .
$$


In stationary case for characteristic length of a relaxation the formula is true

$$
L_{F}=\left(D_{F} \tau_{F}\right)^{1 / 2} \approx 2.64 \times 10^{8} \mathrm{~cm}
$$

Let's assume that in the spatial area $|\boldsymbol{r}| \in\left[0, r_{0}\right]$ in the time moment $t=0$ the fluctuation of chemical potential

$$
F_{1}=\gamma\left(p_{F}^{2} / 2 m\right), \gamma=\text { const, }|\gamma| \ll 1
$$

has occurred. The positive $\gamma$ correspond to productions of plasma quasiparticles pairs and the negative $\gamma$ correspond to annihilations of quasiparticles pairs. Thus for $t \geq 0$ we will obtain

$$
\begin{gathered}
F_{1}(t, \boldsymbol{r})=\gamma\left(p_{F}^{2} / 2 m\right) \Phi_{1}(t, x) \Phi_{1}(t, y) \Phi_{1}(t, z) \exp \{-5 H t\}, \\
\Phi_{1}(t, \xi)=(1 / 2)\left[\operatorname{erf}\left(\left(r_{0}-\xi\right) /\left(4 D_{F} t\right)^{1 / 2}\right)+\operatorname{erf}\left(\left(r_{0}+\xi\right) /\left(4 D_{F} t\right)^{1 / 2}\right)\right], \xi=x, y, z,
\end{gathered}
$$

where

$$
\operatorname{erf}(\zeta)=2 \pi^{-1 / 2} \int_{0}^{\zeta} \exp \left(-\zeta^{\prime 2}\right) \mathrm{d} \zeta^{\prime}
$$

is the error function. From these expressions for velocity $u_{x}(t, x)$ and acceleration of fluctuations flow $a_{x}(t, x)$ along the line $y=0, z=0$ we will obtain the formulas

$$
\begin{aligned}
& u_{x}(t, x)=-(\tau / m) \partial F_{1} / \partial x \\
& =6 \gamma\left(D_{F} / \pi t\right)^{1 / 2} \operatorname{sh}\left(x r_{0} / 2 D_{F} t\right) \Phi_{1}(t, 0)^{2} \exp \left\{-5 H t-\left(r_{0}^{2}+x^{2}\right) / 4 D_{F} t\right\}, \\
& a_{x}(t, x)=\partial u_{x}(t, x) / \partial t \\
& =-3 \gamma\left(D_{F} / \pi\right)^{1 / 2} t^{-3 / 2}\left\{\left[1+10 H t-\left(\left(r_{0}^{2}+x^{2}\right) / 2 D_{F} t\right) \operatorname{sh}\left(x r_{0} / 2 D_{F} t\right)\right.\right. \\
& \left.+\left(x r_{0} / D_{F} t\right) \operatorname{ch}\left(x r_{0} / 2 D_{F} t\right)\right] \Phi_{1}(t, 0)^{2}+2 r_{0}\left(\pi D_{F} t\right)^{-1 / 2} \exp \left\{-r_{0}^{2} / 4 D_{F} t\right\} \\
& \left.\quad \times \operatorname{sh}\left(x r_{0} / 2 D_{F} t\right) \Phi_{1}(t, 0)\right\} \exp \left\{-5 H t-\left(r_{0}^{2}+x^{2}\right) / 4 D_{F} t\right\} .
\end{aligned}
$$

Let's estimate $a_{x}(t, x)$ in the case

$$
t \sim \tau_{p}=2 m \hbar / p_{F}^{2}, x-r_{0} \sim r_{p}=\hbar / p_{F}, r_{0} \gg r_{p} .
$$

As the result we will obtain

$$
\begin{gathered}
a_{x}\left(\tau_{p}, r_{0}+r_{p}\right) \approx\left(\gamma\left|\rho^{(\Lambda)}\right| / m\right)(\hbar / m)^{2} A, A=(5 / 32)(3 \pi)^{3 / 2} \exp \{-3 / 16\}, \\
a_{x}\left(\tau_{p}, r_{0}+r_{p}\right) \approx \gamma \times 3.99 \times 10^{-2} \mathrm{~cm} / \mathrm{s}^{2} .
\end{gathered}
$$

Absolute value of acceleration $a_{x}\left(\tau_{p}, r_{0}+r_{p}\right)$ is proportional of dark energy density and correlates with absolute value of acceleration $\ddot{a}$ if $|\gamma| \sim 10^{-9}$.

From assumption that supernova explosion is connected with vacuum-like plasma fluctuations and it produced the plasma quasiparticles annihilation follows that $\gamma<0$. It corresponds to accelerated motion of fluctuations to the center of fluctuations area and accelerated motion of fluctuation from external observer. By more complex hypotheses it would be possible to explain the de- 
pendence of acceleration of fluctuations from distance to the observer also. But we have not any reasons for such speculations. There is enough that a vacuum-like plasma fluctuations can lead to the accelerated movement of a source of electromagnetic radiation to the right direction.

In the conclusion of this paragraph we note that in the case of "the big fluctuation" when

$$
F_{1}<-\left(p_{F}^{2} / 2 m\right)
$$

Equation (40) becomes unstable. Its solutions increase with increase of time. This situation needs the special research outside the frameworks of this article.

\section{Conclusions}

Presented theoretical results demonstrate that the expansion of the Universe must lead to vacuum-like electron-positron plasma generation. This plasma may exist under the condition of violated chemical equilibrium between electron gas and positron gas only. The difference between chemical potentials of electrons and of positrons describes the level of plasma non-equilibrium. This difference turns out to be proportional to the Hubble's constant and inversely proportional to the plasma temperature in two degrees.

Researched plasma is the material medium that consists of the electron gas, positron gas and a random electromagnetic field caused by transitions between different possible states of particles and antiparticles. Under special conditions, the absolute value of the energy density of random electromagnetic field is more than energy densities of electron gas and positron gas. In this case, the components of plasma acquire the collective properties different from the properties of an ideal gas.

The probability of two-photon annihilation of quasiparticles in this vacuum-like medium is nine times less than for free electrons and positrons. Due to this circumstance, the absolute value of plasma energy density is equal to the density of dark energy obtained as a result of interpretation of the astrophysical measurements. In researched model, the density of dark energy relates with electron mass, electron charge and Hubble constant by very simple formula

$$
\left|\rho^{(\Lambda)}\right|=\left(H / \pi e^{4}\right)(3 m c)^{3} .
$$

The cause of decreasing of the annihilation probability is the attraction that created by a random electromagnetic interaction between particles with the identical sign of the electric charge. The region of the prevalence of Coulomb repulsion is reduced in three times due to this attraction.

Between particle and antiparticle having electric charges of different signs, the random electromagnetic interaction creates the repulsion. It doesn't exert a noticeable impact on the probability of annihilation. However, under the huge distances between an electron and a positron which is about $10^{21} \mathrm{~cm}$, this repulsion exceeds a Coulomb attraction.

Thus, two spatial scales characterize the considered vacuum-like environment: 
repulsion $\left(r_{\text {run }}\right)$ and attraction $\left(r_{e q}\right)$. They differ by thirty-four orders $\left(r_{\text {run }} / r_{\text {eq }} \sim 10^{34}\right)$.

Analyses of small fluctuations in vacuum-like plasma demonstrated that acceleration of such fluctuations may be very big in time period about $\tau_{p} \approx 4.52 \mathrm{~s}$. It means that vacuum-like plasma fluctuations can lead to the accelerated movement of a source of electromagnetic radiation. Their influence on the radiation of supernova Ia type demands the additional research. It is well known that interaction between cosmic objects and electron-positron plasma may produce very interesting phenomena [18].

\section{References}

[1] Obukhov, I.A. (2016) Cosmological Constant and Energy Density of Random Electromagnetic Field. Journal of High Energy Physics, Gravitation and Cosmology, 2, 312-319. https://doi.org/10.4236/jhepgc.2016.23028

[2] Gliner, E.B. (1966) Algebraic Properties of the Energy-Momentum Tensor and Vacuum-Like States of Matter. Soviet Physics-JETP, 22, 378-382. http://www.jetp.ac.ru/cgi-bin/r/index/e/22/2/p378?a=list

[3] Zel'dovich, Ya.B. (1968) The Cosmological Constant and the Theory of Elementary Particles. Soviet Physics Uspekhi, 11, 381-393.

http://ufn.ru/ru/articles/1968/5/m/ https://doi.org/10.1070/PU1968v011n03ABEH003927

[4] de Groot, S.R., van Leeuwen, W.A. and van Weert, Ch.G. (1980) Relativistic Kinetic Theory: Principles and Applications. North-Holland Publishing Company, Amsterdam, New York and Oxford.

[5] Petrov, N. and Brankov, Y. (1986) Modern Problems of Thermodynamics. Mir, Moscow. (In Russian)

[6] Obukhov, I.A. (2014) Nonequilibrium Effects in One-Dimensional Quantum Devices. Lambert Academic Publishing, Saarbrücken.

[7] Edwards, S.F. (1958) A New Method for the Evaluation of Electric Conductivity in Metals. Philosophical Magazine, 3, 1020-1031. https://doi.org/10.1080/14786435808243244

[8] Dirac, P.A.M. (1930) On the Annihilation of Electrons and Protons. Mathematical Proceedings of the Cambridge Philosophical Society, XXVI, 361-375. https://doi.org/10.1017/S0305004100016091

[9] Chernin, A.D. (2013) Dark Energy in Systems of Galaxies. JETP Letters, 98, 353-364. http://www.jetpletters.ac.ru/ps/2018/article_30426.pdf https://doi.org/10.1134/S002136401319003X

[10] Berestetskii, V.B., Pitaevskii, L.P. and Lifshitz, E.M. (1982) Quantum Electrodynamics. 2nd Edition, Butterworth-Heinemann, Oxford.

[11] Lifshitz, E.M. and Pitaevskii, L.P. (1980) Statistical Physics, Part 2: Theory of the Condensed State. Pergamon Press.

[12] Umezawa, H., Matsumoto, H. and Tachiki, M. (1982) Thermo Field Dynamics and Condensed States. North-Holland Publishing Company, Amsterdam, New York and Oxford.

[13] Alves, M.E.S., Miranda, O.D. and de Araujo, J.C.N. (2010) Can Massive Gravitons Be an Alternative to Dark Energy? 
[14] Hinshaw, G., Larson, D., Komatsu, E., et al. (2013) Nine-Year Wilkinson Microwave Anisotropy Probe (WMAP) Observations: Cosmological Parameter Results. https://lambda.gsfc.nasa.gov/product/map/dr5/pub_papers/nineyear/cosmology/w map_9yr_cosmology_results.pdf

[15] Tangherlini, F.R. (2017) A Possible Alternative to the Accelerating Universe IV. Journal of Modern Physics, 8, 622-635. https://doi.org/10.4236/jmp.2017.84042

[16] Corda, C. (2009) Interferometric Detection of Gravitational Waves: The Definitive Test for General Relativity. International Journal of Modern Physics D, 18, 2275-2282. https://doi.org/10.1142/S0218271809015904

[17] Frontera1, F., Guidorzi, C., Montanari, E., et al. (2009) The Gamma-Ray Burst Catalog Obtained with the Gamma-Ray Burst Monitor aboard Beppo SAX. The Astrophysical Journal Supplement Series, 180, 92-223.

http://iopscience.iop.org/article/10.1088/0067-0049/180/1/192/pdf

[18] Gaina, A. (1995) Black Holes as Electric Condensers. Romanian Astronomical Journal, 5, 131-134. 